\title{
Médiévales
}

Langues, Textes, Histoire

66 | printemps 2014

Harmonie Disharmonie

\section{Anne-Zoé RILLON-MARNE, "Homo considera ». La Pastorale lyrique de Philippe le Chancelier. Une étude des conduits monodiques}

Turnhout, Brepols («Studia artistarum. Études sur la Faculté des arts dans les universités médiévales », 34), 2012, 370 p.

\section{(2) OpenEdition}

Journals

Édition électronique

URL : https://journals.openedition.org/medievales/7300

DOI : $10.4000 /$ medievales.7300

ISSN : $1777-5892$

Éditeur

Presses universitaires de Vincennes

Édition imprimée

Date de publication : 30 juin 2014

Pagination : 208-211

ISBN : 978-2-84292-405-8

ISSN : 0751-2708

Référence électronique

Martine Clouzot, "Anne-Zoé Rillon-mARne, « Homo considera ». La Pastorale lyrique de Philippe le Chancelier. Une étude des conduits monodiques », Médiévales [En ligne], 66 | printemps 2014, mis en ligne le 10 juillet 2014, consulté le 22 avril 2022. URL : http://journals.openedition.org/medievales/ 7300 ; DOI : https://doi.org/10.4000/medievales.7300

Ce document a été généré automatiquement le 22 avril 2022.

Tous droits réservés 


\section{Anne-Zoé RILLON-MARNE, « Homo considera ». La Pastorale lyrique de Philippe le Chancelier. Une étude des conduits monodiques}

Turnhout, Brepols («Studia artistarum. Études sur la Faculté des arts dans les universités médiévales », 34), 2012, 370 p.

\section{Martine Clouzot}

\section{RÉFÉRENCE}

Anne-Zoé RILLON-MARNE, «Homo considera ». La Pastorale lyrique de Philippe le Chancelier. Une étude des conduits monodiques, Turnhout, Brepols ( $«$ Studia artistarum. Études sur la Faculté des arts dans les universités médiévales », 34), 2012, 370 p.

L'ouvrage d'A.-Z. Rillon-Marne est issu de sa thèse de doctorat de musicologie médiévale dirigée par 0. Cullin, Professeur à l'Université de Tours. Il est consacré à l'étude des conduits monodiques composés par Philippe le Chancelier pour les chantres de la cathédrale Notre-Dame de Paris au XIII ${ }^{\mathrm{e}}$ siècle. Mettant en relation la prédication et la composition musicale, l'auteure a analysé comment le Chancelier a mêlé ces deux pratiques pour les transformer en stratégies de communication morale et sonore très élaborées. Elle a voulu montrer la subtilité « des pratiques culturelles communes aux sermons et aux compositions musicales ", dont le but commun est de convaincre, comme le rappelle 0 . Cullin dans sa préface, et étudier «au plus près les pratiques musicales en vogue dans les cercles ecclésiastiques de la cathédrale de Paris entre les dernières années du XII ${ }^{e}$ siècle et 1236 , date de la mort de l'auteur ». Il faut saluer ce travail de recherche très fouillé, tant au niveau de la constitution (difficile) du corpus que de la mise en contexte et de l'analyse méthodologique proprement dite des conduits monodiques du Chancelier. L'auteure a aussi le mérite d'être ouverte à 
d'autres disciplines, telles la littérature et l'histoire. Aussi lui reprochera-t-on, avec bienveillance, certaines questions inutiles et assez typiques de la musicologie médiévale, car la qualité de son travail montre qu'elle les a dépassées par le renouvellement de son approche. Par exemple, le paradoxe du théologien qui est aussi poète n'a pas vraiment lieu d'être soulevé. En effet, en replaçant la production musicale du Chancelier dans son contexte culturel, comme l'auteure l'a très bien fait, il est rappelé que les plus grands «intellectuels » étaient formés à différentes formes d'expression savante. Abélard, théologien et compositeur de chansons et d'hymnes, comme l'a montré $\mathrm{M}$. Clanchy, en est un exemple caractéristique.

2 L'ouvrage est composé de huit chapitres allant de la présentation des sources du corpus à la formation d'une pastorale musicale par le Chancelier, en passant par la rhétorique poétique et musicale et la prédication morale de ses conduits. Fort utiles, plusieurs tableaux récapitulatifs ont été intégrés dans les chapitres. Quant aux annexes, elles sont très complètes, comprenant les transcriptions musicales de vingt conduits monodiques en latin accompagnées d'une fiche de présentation (p. 261-338), ainsi qu'un glossaire médiéval, une bibliographie spécialisée, deux index des noms médiévaux et compositions cités.

3 Sous la plume des philologues et historiens du XIXe siècle, l'historiographie sur Philippe le Chancelier a d'abord mal jugé le personnage et sa production musicale. Longtemps, il a été confondu avec un certain Philippe de Grève, jusqu'à ce que H. Meylan en 1927 distingue les deux figures. Puis, la réévaluation du théologien a été amorcée par P. Meyer en 1864 et d'autres. Leur intérêt pour l'édition des grandes sommes scolastiques du xIII ${ }^{e}$ siècle a favorisé la « réhabilitation » du Chancelier dans le domaine de la théologie. Parallèlement, les sermons du prédicateur ont fait l'objet du seul travail de synthèse réalisé aujourd'hui, grâce à l'édition du répertoire de J. B. Schneyer (1962). Enfin, le corpus poético-musical de l'époque étant bien constitué, la participation du poète au répertoire de son temps a suscité l'intérêt des musicologues et plusieurs éditions musicologiques. Le corpus poétique a également été étudié grâce à $\mathrm{P}$. Dronke (1987). Cependant, c'est par le travail de recherche méticuleux de T. B. Payne (1991, 2001) que Philippe le Chancelier et sa musique ont pu être placés au même rang que les maîtres de Notre-Dame, Léonin et Pérotin. Payne a pu mettre en évidence le caractère novateur de sept motets et compléter le corpus de vingt-sept compositions étonnantes par leur diversité formelle et stylistique. Quant aux conduits monodiques, leur analyse est un domaine récent de la recherche musicologique (S. Rankin) à laquelle A.-Z. RillonMarne a choisi de contribuer à partir des conduits du Chancelier. Elle rappelle avant tout que le terme conductus est difficile à définir car ses aspects sont variés depuis son apparition dans les manuscrits de Saint-Martial de Limoges. Le seul point établi est le texte en latin versifié, et donc les dispositifs sonores structurant le texte poétique et la mélodie par l'échelle modale.

4 Malgré les difficultés d'attribution, trois collections musicales et poétiques ont été attribuées au Chancelier (Londres, Prague, Darmstadt). Elles ont été confirmées par des témoignages narratifs, dont le Dit du chancelier Philippe par son ami et protecteur Henri d'Andeli, qui présente le chancelier en trouvère et joueur de vièle : la transfiguration du théologien en « jongleur de Dieu » appartient à la littérature mariale de l'époque et confirme les rapports ambivalents entre les clercs et les jongleurs - les articles de J. Baldwin et de M. Zink sur «le Chancelier jongleur de Dieu» auraient pu être mentionnés en notes de bas-de-page. Il est un peu étonnant que l'auteure cherche à 
confirmer le sérieux de l'activité musicale du Chancelier (p. 70), car, évidemment, celleci est sérieuse à n'en pas douter, et il est dommage que les clivages disciplinaires laissent planer une certaine suspicion sur sa qualité et suscitent encore ce genre de critique. D'autres productions musicales attestent de la participation du Chancelier à leur constitution: certaines compositions monodiques du corpus de Notre-Dame; six conduits sur les deux cent vingt-huit compositions du manuscrit des Carmina Burana; et quatorze insertions musicales dans le Roman de Fauvel (Paris, BnF, fr. 146) montrant la réception en 1314 des monodies composées dans les années 1170. Plutôt que de " mouvements littéraires contestataires" (p. 77), il serait plus approprié de parler de critique morale et de satire politique dont seuls les clercs peuvent faire preuve au tournant du XIII ${ }^{e}$ siècle. Enfin, A.-Z. Rillon-Marne mentionne une collection composite de huit compositions contenues dans un livre liturgique (Rome).

Le lien entre le corpus musical et la prédication morale est établi dans le troisième chapitre «Un corpus moralisateur». A.-Z. Rillon-Marne le justifie parfaitement en rappelant que le Chancelier est théologien et prédicateur et " possède des compétences oratoires, dialectiques, didactiques, juridiques ». Le fait que le texte latin précède la composition musicale montre les imbrications intrinsèques de la poésie et de la mélodie dans le processus de création. Les liens structurels entre texte et musique donnent aux conduits toute leur efficacité communicative. Le but du prédicateur visant la conversion des mœurs, les discours sur le Bien et le Mal, les vices et les vertus, la vanité, la fragilité humaine, la fuite du temps, le contemptus mundi s'adressent aux laïcs et aux clercs, ceux-ci étant particulièrement visés par les vices de l'avarice, l'appât du gain, l'hypocrisie et la luxure. Cette entreprise de moralisation musicale et orale est caractérisée par différents procédés littéraires de la communication, issus des traités oratoires romains.

6 Les chapitres suivants présentent des études de cas très précises et tout à fait convaincantes sur la rhétorique poétique (répétitions, jeux de mots) et musicale (mélodie, repetitio, gradatio) des conduits. Ils font la démonstration de la maîtrise de la rhétorique poétique du Chancelier avec laquelle il a su structurer le texte et à organiser avec virtuosité les colores sonores des mots. Charmant les oreilles et frappant les esprits, cette communication est morale car elle est mentale : elle organise la pensée et suscite la méditation. Basée sur une rhétorique de l'oralité, la construction des conduits est, d'après l'auteure, semblable à celle des sermons (cinquième chapitre) dans leur dimension sonore. Citant Guido d'Arezzo et s'appuyant sur le livre majeur de L. Treitler - With Voice and Pen (2003) -, A.-Z. Rillon-Marne rappelle les liens d'analogie entre le langage et la mélodie. Avec des analyses musicologiques très élaborées sur les strophes, les vers, la figure d'épanadiplose (la correspondance entre le début et la fin d'un conduit), elle arrive à la définition convaincante du "conductus comme discours poétique et moral» (chapitre 6) : le conduit est à la fois un tout unifié et fragmenté, qui doit être organisé «selon une logique et une cohérence au niveau de la macrostructure» (p. 196), afin d'être saisi par la mémoire de l'auditeur. Se basant sur les recherches fondamentales de F. Reckow, A.-Z. Rillon-Marne peut préciser la définition du conduit, qui « est la mise en œuvre d'un processus, une progression dynamique dans le temps et l'audition", ménageant une tension sonore culminante (climax) généralement au milieu de la composition. Le parallèle avec la notion de ductus analysée par M. Carruthers vient fort à propos. La démonstration est renforcée par les analyses de deux conduits dans le septième chapitre. 
7 Quant au huitième et dernier chapitre, il témoigne d'une réflexion plus personnelle de l'auteure, attentive au contexte de production et de performance des conduits du Chancelier. Dépassant la notion isolée et anachronique de "genre musical», comme il est courant en musicologie médiévale, elle a préféré s'attacher à déterminer « comment une partie des conduits évolue dans une communauté de savoir-faire, la rhétorique du discours que l'auteur a apprise, entre autres, par la pratique de la prédication" (p. 239). Elle peut alors conclure que «le conduit est conçu pour la performance, pour agir donc pour être perçu » par les sens et l'intelligence des auditeurs. Par ses sermons et ses compositions musicales, Philippe le Chancelier, théologien, poète et compositeur, a ainsi pleinement œuvré, à Paris, à la réforme spirituelle et morale de la société de son temps.

8 L'ouvrage d'A.-Z. Rillon-Marne offre une étude de cas très réussie autour d'une figure majeure du XIII siècle, Philippe le Chancelier, et de ses compositions poétiques. On continuera à lire ses travaux avec grand intérêt. 